\title{
On the use of WiMAX and Wi-Fi to provide in-vehicle connectivity and media distribution
}

\author{
Lerotholi S. Mojela and Marthinus J. Booysen \\ Department of Electrical and Electronic Engineering and the MIH Media Lab, Stellenbosch University \\ Stellenbosch, South Africa. Email: mjbooysen@sun.ac.za
}

\begin{abstract}
The recent emergence of ubiquitous wireless connectivity and the increasing computational capacity of modern vehicles have triggered immense interest in the possibilities of vehicular connectivity. A plethora of potential applications for vehicular networks have been proposed in the areas of safety, traffic infrastructure management, information, and entertainment. The broad range of applications requires creative utilization of the available wireless medium, using a combination of existing and novel wireless technologies. In this paper we evaluate one such configuration, assuming dedicated short range communication for safety applications, and using Wi-Fi and WiMAX for non-safety applications. Little is known about the media streaming performance of these wireless technologies in realistic vehicular ad-hoc network (VANET) scenarios. Due to the extreme mobility and unpredictable environmental aspects in a real road environment, we perform and present an empirical evaluation. We evaluate a multi-vehicle to infrastructure (V2V2I) VANET, using Wi-Fi for the vehicle-to-vehicle communication and WiMAX for the vehicle to infrastructure (V2I) communication.
\end{abstract}

Index Terms-VANET, vehicular-to-vehicle, vehicle-toinfrastructure, WiMAX, Wi-Fi.

\section{INTRODUCTION}

Vehicular ad-hoc networks (VANETs) are formed by moving vehicles and stationary road side units (RSUs) equipped with short range wireless communication devices. In these wireless networks, vehicles communicate with each other, called vehicle-to-vehicle (V2V) communication, and with infrastructure networks, called vehicle-to-infrastructure(V2I) communication, or a hybrid of these two architectures, called V2V2I communication[1, 2, 3].

The development and deployment of Intelligent Transportation Systems (ITS) has been the primary driver for the research on inter-vehicular communications [4], aiming to minimize accidents and improve traffic conditions by providing vehicles, drivers and passengers with information otherwise not available. A number of applications have been proposed for VANETs, broadly classified into safety and non-safety applications [5, 6]. Safety applications convey safety critical information based on sensor data from other vehicles or RSUs to report and avoid emergencies [1,5]. Examples include a sudden brake warning sent from a remote preceding car, information about road conditions and maintenance, and accident annunciations. Non-safety applications include entertainment and online connectivity, which have received little attention in the literature, and general traffic management $[1,7,8]$. The non-safety applications typically obtain data on-demand such that a node requests information of interest $[1,6]$.
Examples of these are electronic payments, file sharing, and audio or video streaming. A key aspect of these commercial applications is the availability of high bandwidth and stable Internet connectivity.

The nodes in VANETs, unlike those in MANETs are characterized by high mobility and sudden speed and direction changes, which lead to rapid network topology changes [1, 9]. These factors impose a number of challenges, such as message routing techniques as communication links are short lived, signal degradation and multipath fading, and Medium Access Control (MAC) of the shared wireless medium [10]. An important factor is the prioritization of messages when sharing a single network medium; safety applications should have higher priority, but sometimes non-safety applications could already have flooded the network causing delay of critical messages. One way to overcome these challenges is to separate the applications by applying a cross layer architecture, as shown in Fig. 1 [11].

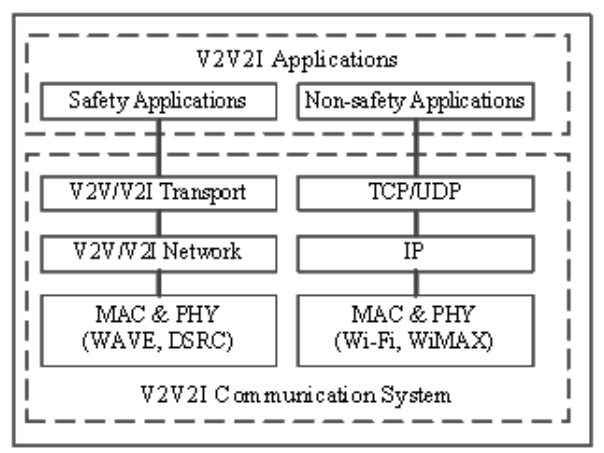

Fig. 1: V2V2I system components and functionality.

The recently introduced IEEE $802.11 \mathrm{p}$, which forms part of Wireless Access in the Vehicular Environment (WAVE) [12, 14], could be used for safety applications, while the existing standards, for example IEEE $802.11 \mathrm{~g} / \mathrm{n}$ (Wi-Fi) and IEEE 802.16 (WiMAX), could be used for non-safety applications. This architecture will improve overall system performance and more efficiently utilize resources [11]. Wi-Fi is a short range $(+/-200 \mathrm{~m})$ wireless local area network (WLAN) technology protocol based on the IEEE 802.11 network standard [13] operating on an unlicensed radio frequency of $2.4 \mathrm{GHz}$ offering high data rates of up to $150 \mathrm{Mbps}$ (IEEE 802.11n). WiMAX is a long range $(50 \mathrm{~km})$ wireless metropolitan area network (WMAN) technology based on the IEEE 802.16 standard [15] 
operating on both licensed, 10-66 GHz and unlicensed, 5-6 $\mathrm{GHz}$ radio frequencies.

The development of VANET applications and protocols require a thorough understanding of how networks will perform under realistic VANET environments. Quantitative aspects of connection performance under various movement and environmental conditions include:

- Duration of a typical communication link,

- Amount of data that can be transferred during a contact period

- Instantaneous throughput while the link is active, and

- Amount of jitter evident.

\section{A. Contributions of work}

Our research empirically examines the performance of a simple VANET that uses Wi-Fi and WiMAX to realize a V2V2I network architecture, as indicated in Fig. 2. For our experiment Wi-Fi is used for V2V communications and WiMAX for V2I communications. We focus on non-safety applications including Internet connectivity and media streaming in built and unpopulated urban environments. Wi-Fi performance for $\mathrm{V} 2 \mathrm{~V}$ communication is also investigated in a highway environment. Our results show that the network architecture employed provides a robust and functional channel for V2V, V2I, and V2V2I data delivery under certain scenarios.

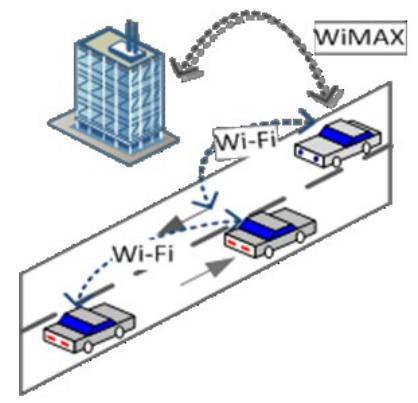

Fig. 2: V2V2I setup for vehicles crossing and following.

The remainder of this paper is organized as follows, section II gives an overview of related work, section III describes our experimental setup, and section IV presents the results and analysis. Section V concludes the paper.

\section{RELATED WORK}

The most adopted vehicular networking architectures consist of Road-Side Units (RSUs) existing along the road and vehicle equipment called On-Board Units (OBUs). OBUs and RSUs form ad-hoc networks where communication takes place directly between OBUs via multi-hop or single-hop (called $\mathrm{V} 2 \mathrm{~V}$ ), or in which OBUs communicate with RSUs in order to connect to external networks or infrastructure (called V2I) and where OBUs communicate with the RSUs through other OBUs (called V2V2I) [3, 16, 17, 18, 19].

In [20], Tufail et al. studied the behavior of network connections that are initiated over an IEEE 802.11g channel from a moving car. The goal was to investigate and discuss the possibility of using IEEE 802.11 as the protocol to establish connection between fast moving vehicles and to understand the impact of the car's speed. The experiment involved measuring the amount of data transferred during the short spurts the connection was intact. This involved two vehicles moving in opposite directions at the same constant speed, though for each test the speed was changed. They showed that as the speed increases the intact time decreases so does the data rate hence less data can be transferred. They achieved a peak data rate of $7.7 \mathrm{Mbps}$ at the speed of $20 \mathrm{mph}$ or $32 \mathrm{~km} / \mathrm{h}$ (relative speed of $40 \mathrm{mph}$ or $64 \mathrm{~km} / \mathrm{h}$ ) and managed to transfer 15.1 MB. At the speed of $60 \mathrm{mph}$ or $96 \mathrm{~km} / \mathrm{h}$ (relative speed of $120 \mathrm{mph}$ or $193 \mathrm{~km} / \mathrm{h}$ ), it was possible to transfer $0.3 \mathrm{MB}$ of data and maintain a data rate of about 1 Mbps. Marcelo et al. [2] had a test-bed similar to [20]. They investigated the characteristics of links formed by in-car nodes running off the shelf wireless technologies, both IEEE 802.11a and IEEE $802.11 \mathrm{~g}$, in ad hoc mode. They also included the impact of transport protocols (TCP and UDP) and packet size on the amount of data transferred. The car speed was varied from $20 \mathrm{~km} / \mathrm{h}$ to $60 \mathrm{~km} / \mathrm{h}$ (relative speed of $40 \mathrm{~km} / \mathrm{h}$ to $120 \mathrm{~km} / \mathrm{h}$ ), while the packet sizes tested were 150,500 and 1460 bytes for both TCP and UDP. The results show that using TCP instead of UDP reduces the average total amount of data transferred, and as speed increased no data was received due to the long time TCP spends trying to establish a connection. They also showed that higher frequency $5.15 \mathrm{GHz}$ IEEE 802.11a is more prone to propagation problems (diffraction, reflection, and absorption) than lower frequency IEEE $802.11 \mathrm{~g}$ which lead to shorter contact time. Again they showed a trade-off between speed and packet size; decreasing the packet size reduced the capacity loss and increased the capacity as speed increased.

Wellens et al. [21] investigated the one hop performance of IEEE $802.11 \mathrm{a} / \mathrm{b} / \mathrm{g}$ (AP) for Car to Car (C2C) and Car to Roadside (C2R) scenarios. They had one node operating as an Access Point (AP) while the other was in normal configuration. For $\mathrm{C} 2 \mathrm{R}$ measurements, they placed the $\mathrm{AP}$ in the middle of a $2 \mathrm{~km}$ road while a car connects to it as it passes by. Both UDP and TCP traffic with different packet sizes were generated to evaluate their effect at vehicular speeds and these were compared with the static measurement values performed in the lab where the distance between AP and client node was $1 \mathrm{~m}$. The tests were conducted under urban scenario with the presence of traffic and tall buildings and on a highway. Throughput, signal to noise ratio (SNR), and frame error rate were monitored in these experiments. They found throughput at $120 \mathrm{~km} / \mathrm{h}$ to be slightly lower than that of the static case, and larger packets presented a worse throughput compared to smaller packets. The communication range was also found to be larger when using lower physical layer rates due to the use of less aggressive coding and modulation schemes. Speed did not affect the performance of Wi-Fi but distance and availability of line of site did create a negative impact.

Chou et al. [22] studied the feasibility of using fixed WiMAX (IEEE 802.16d) vs. Wi-Fi (IEEE 802.11g) for V2I communications. The measurements focused on through- 
put, latency and packet loss. They found that even though they managed to achieve data transfers at long ranges with WiMAX, with Wi-Fi one can get higher throughput and a shorter latency at shorter distances. They also found that larger frame sizes offer better throughput but at a cost of longer delays. The same study was conducted on QualNet 4.5 simulation tool by Msadaa et al. [23], but they used IEEE 802.11p and mobile WiMAX (IEEE 802.16e). They evaluated the performance for different vehicle speed, data rates and network deployments. They also showed that mobile WiMAX suffers longer delays compared to IEEE 802.11p and the average throughput does not depend on the vehicle speed.

J. Eriksson et al. [24] designed, implemented and experimented evaluation of Cabernet Transport Protocol (CTP), a content delivery network for vehicles moving in and around cities. Cabernet delivers data to and from cars using open IEEE $802.11 \mathrm{~b} / \mathrm{g}$ access points (APs) that the cars connect to, opportunistically while they travel. The primary goal of CTP was to develop techniques that allow moving cars to obtain high data transfer throughput through these APs. The system was deployed in 10 taxis running in the Boston area. The nodes/vehicles were running QuickWiFi and the system running CTP. CTP achieved double the throughput of TCP over paths with high non-congestion losses with a mean throughput of $800 \mathrm{kbps}$ when connectivity is present. In an end-to-end performance evaluation, Cabernet was able to achieve an end-to-end throughput of $86.5 \mathrm{kbps}$ per car during a trip.

\section{EXPERIMENTAL SETUP}

We investigate the performance of using a combination of WiMAX and Wi-Fi to respectively provide V2I and V2V connectivity in a V2V2I vehicular network. Our experiment consists of two vehicles (mobile nodes) that are linked with an ad-hoc Wi-Fi connection and a stationary base station with a dedicated WiMAX connection to one of the vehicles. The setup can be seen in Fig. 2. The experimental setup was designed to accurately reflect conditions present in an urbanized environment. In this work we focus on scenarios where vehicles travel in the same direction (following) and in opposite directions (crossing). The experiments were carried out on the Stellenbosch University campus on two routes within range of the WiMAX Base Station (BS). The legal speed limit on these routes is $60 \mathrm{~km} / \mathrm{h}$. The first route is close to the BS in an open area with direct Line Of Sight (LOS) of the BS, while the second route is further from the BS in the city center, a built environment representing non line of sight (NLOS). Since the experiment concurrently uses two different wireless technologies, initial tests were performed to characterize the individual performance of each technology before combining the two. We therefore evaluated Wi-Fi and WiMAX performance separately on both routes and these tests are depicted in Figure 3.

For Wi-Fi only communications (V2V), two tests were carried out: Vehicles following each other on the routes and vehicles crossing each other (from opposite directions) on

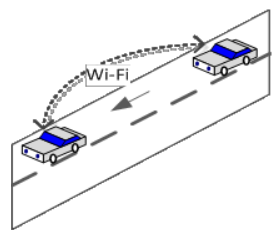

(a) V2V following.

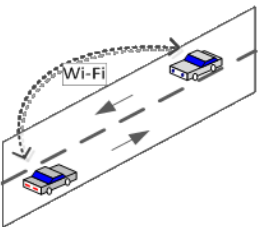

(b) V2V crossing.

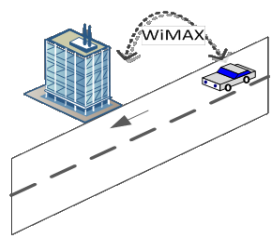

(c) V2I
Fig. 3: Initial performance tests.

both routes, depicted in Fig. 3a and Fig.3b respectively. For WiMAX only communications (V2I), the WiMAX enabled vehicle was driven along both routes, Fig. 3c. For the complete experiment, using V2V2I communications, the two tests, following and crossing, were repeated on both routes. An additional test was also performed where the two vehicles were following each other for $50 \mathrm{~km}$ on a highway route with speeds up to $120 \mathrm{~km} / \mathrm{h}$ and the separation range kept below 300 meters. For this test, only the Wi-Fi connection was active. In addition to the quantitative link performance results, the V2I and V2V2I configuration was also used to qualitatively evaluate the link using video and audio firstly streaming between the vehicles, secondly from the base station to the WiMAX enables vehicle, and thirdly from the BS through the WiMAX enabled vehicle to the other vehicles.

Each vehicle was equipped with a laptop with an external Wi-Fi adapter. The adapters were mounted outside the vehicles to provide LOS and increase coverage. The WiMAX BS was mounted on a five-story building. In addition, both the laptops had a GPS dongle to monitor the position and speed of the vehicles. Speed (relative speed), separation (between vehicles and from BS), signal strength (WiMAX and WiFi), modulation type (WiMAX and Wi-Fi), throughput, data transferred, contact time (time from first packet to last packet received), and jitter were recorded for each of the experiments conducted.

\section{RESUlTS AND DisCUSSION}

In this paper we evaluate the performance of $\mathrm{Wi}-\mathrm{Fi}$ (IEEE 802.11n) and WiMAX (IEEE 802.16d) for different VANET architectures based on measurements of bandwidth, received signal strength, jitter, and total data transferred. Signal strength, herein referred to as received signal strength indicator (RSSI) is measured in decibels given in relation to one milliwatt $(\mathrm{dBm})$. Throughput is a measure of the amount of data that can be transmitted in a given amount of time measured in kilobits per second (kbps) or Megabits per second (Mbps). Jitter is the delay variation in packets measured in milliseconds ( $\mathrm{ms}$ ) and total data transferred represents the data bytes (B) that are correctly received on the server side and with an acknowledgment correctly received on the client. When this section refers to LOS and NLOS we refer to WiMAX line-ofsight or non-line-of-sight, since Wi-Fi was always in LOS.

The experimental results are graphically presented on time based plots, with time increasing to the right. In order to 


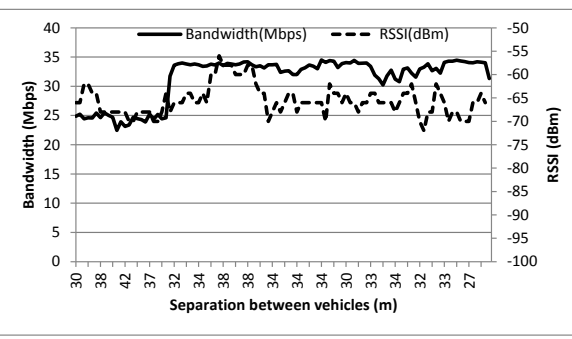

(a) $\mathrm{V} 2 \mathrm{~V}$ vehicles following.

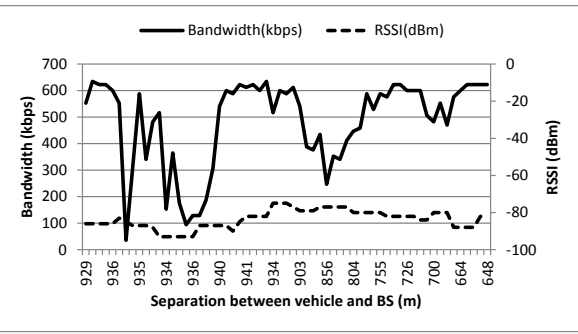

(d) V2I with NLOS.

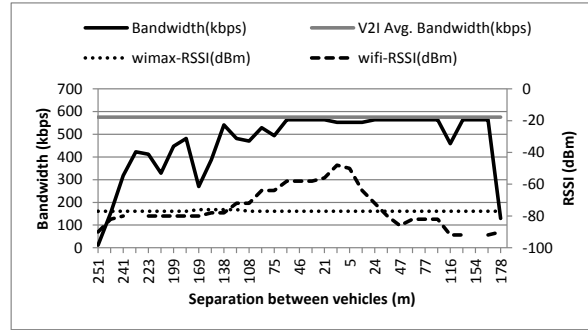

(g) V2V2I crossing with LOS.

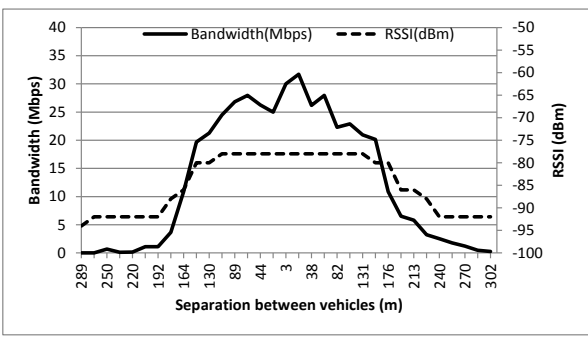

(b) V2V vehicles crossing.

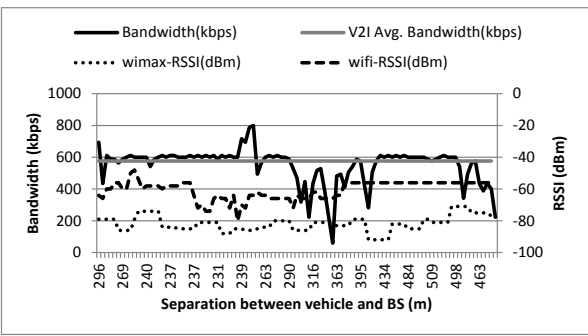

(e) V2V2I following with LOS.

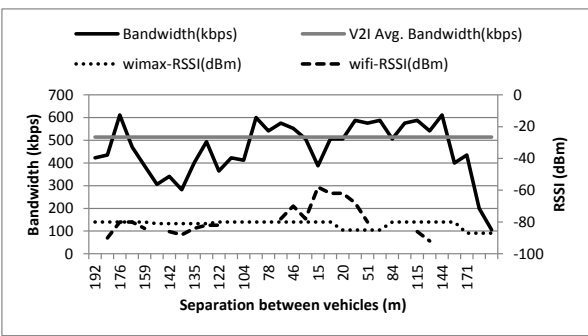

(h) V2V2I crossing with NLOS.

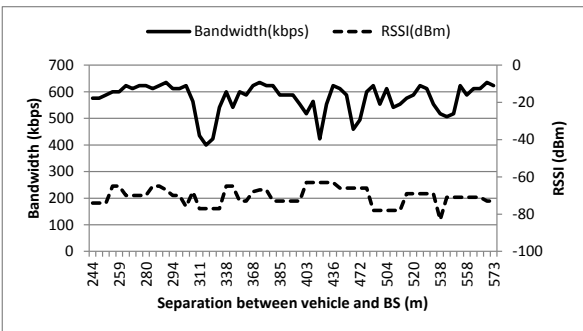

(c) V2I with LOS.

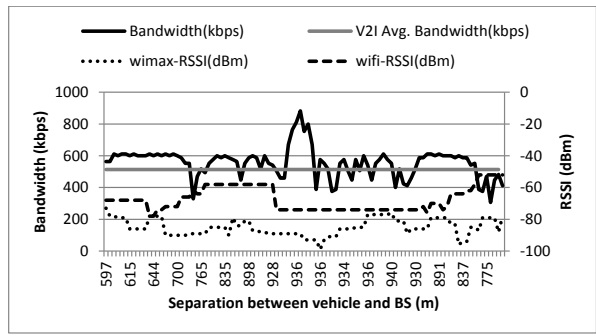

(f) V2V2I following with LOS.

Fig. 4: Initial performance tests.

capture the significance of physical separation, the labels presented on the horizontal axes are separation at the time, rather than the time.

\section{A. Vehicle to vehicle communication (Wi-Fi only)}

Fig. 4a shows the bandwidth and signal strength with respect to separation between the two vehicles following each other. The test was conducted on a highway with a measured average absolute vehicle speed of $113 \mathrm{~km} / \mathrm{h}$. The average results recorded for the tests are separation of $34 \mathrm{~m}$, bandwidth of $31.3 \mathrm{Mbps}$, and jitter of $0.38 \mathrm{~ms}$. A total of $386.3 \mathrm{MB}$ of data was transferred in a representative 100 seconds period. The maximum peak bandwidth recorded was 34.5 Mbps occurring at random points in the test. Equivalent results were obtained when the setup was conducted in an urban environment.

At various stages of the experiment there were obstacles (e.g. other vehicles) in-between and around the two communicating vehicles. This is visible from the graph where the throughput increases and decreases sharply. Since the radio was set to automatically adjust the transmit power, the radio would automatically adjust the power level when the link became weak. The variation in signal strength was therefore additionally affected by the increase in transmission power of the Wi-Fi card.

Fig. 4b shows a graph of the two vehicles traveling in opposite directions at an average relative speed of $64 \mathrm{~km} / \mathrm{h}$ in an urban area. The average contact time recorded was $33 \mathrm{~s}$ and the average maximum communication range was found to be $302 \mathrm{~m}$ with an average bandwidth of $13.7 \mathrm{Mbps}$ per test run taken over the period of established contact, average jitter of $1.88 \mathrm{~ms}$ and an average of $51.7 \mathrm{MB}$ data transferred per contact period. The maximum peak bandwidth of $31.7 \mathrm{Mbps}$ was reached with the vehicles $0 \mathrm{~m}$ from each other, i.e. at the point of crossing. The plot shows the bandwidth and signal strength with respect to separation between the two vehicles. From the figure, the Wi-Fi bandwidth seems to indicate a dependence on signal strength which is in turn affected by the separation between the two vehicles. The same behavior is also reported in $[2,21,22]$, as the two communicating nodes come closer, the signal strength increases, and so does the bandwidth.

\section{B. Vehicle to Infrastructure communication (WiMAX only)}

The WiMAX bandwidth and signal strength in the V2I scenario for LOS and NLOS environments are shown in Figs. $4 \mathrm{c}$ and $4 \mathrm{~d}$ respectively. The results represent an run that lasted 
for 100 seconds. The WiMAX bandwidth drops from around $5 \mathrm{Mbps}$ to around $500 \mathrm{kbps}$ as soon as the vehicle becomes mobile. We determined that the cause of this to be modulation change from 64QAM to either BPSK or QPSK caused by a packet loss of more than $60 \%$.

For the LOS route, the average vehicle speed was $31 \mathrm{~km} / \mathrm{h}$ and a total data transfer of $6.35 \mathrm{MB}$ at an average bandwidth of $521 \mathrm{kbps}$ with an average jitter of $8.22 \mathrm{~ms}$.

For the NLOS route, the average vehicle speed was $33 \mathrm{~km} / \mathrm{h}$. The average bandwidth of $518 \mathrm{kbps}$ was reached and produced a total data transfer of $6.33 \mathrm{MB}$ with an average jitter of 8.56 ms.

The NLOS signal strength is weaker than that of LOS test due to the distance from the base station and the presence of obstacles that affect the signal. This can also be seen from the bandwidth plot: Even though the bandwidth peaks at about the same value as in LOS conditions, in NLOS the fluctuation is higher and the bandwidth drops to close to zero.

\section{Vehicle to Vehicle to Infrastructure (Wi-Fi and WiMAX)}

1) Vehicles following each other: Figs. $4 \mathrm{e}$ and $4 \mathrm{e}$ respectively show the bandwidth and signal strength for vehicles following each other in an urban environment under LOS and NLOS conditions using the V2V2I setup. The average link bandwidth for LOS and NLOS in the V2I only tests is also shown for reference.

For the LOS route, the average absolute vehicle speed was $27 \mathrm{~km} / \mathrm{h}$ with an average vehicle separation of $31 \mathrm{~m}$. This resulted in an average bandwidth of $539 \mathrm{kbps}$ and total data transfer of $6.64 \mathrm{MB}$ in the 100 seconds period. The graph shows a noticeable increase in bandwidth at an intersection where the vehicles had to stop. The temporary cessation has this effect on the bandwidth due to the lower WiMAX packet loss ratio. This event is followed by bandwidth decrease as the distance between the vehicles increased to $150 \mathrm{~m}$ with respect to the second vehicle still at the intersection. But the bandwidth settles to the same value as that of V2I average, showing the performance dependence on WiMAX.

The performance results on the NLOS route for V2V2I are similar to that experienced for V2I as we see similar average bandwidth measurements: The visible difference being the high fluctuation in bandwidth and signal strength. Again a bandwidth increase is noticeable where the vehicles stopped at an intersection. The 100 seconds period at an average separation between the vehicles of $40 \mathrm{~m}$ traveling at an average speed of $25 \mathrm{~km} / \mathrm{h}$ resulted in an average bandwidth of $543 \mathrm{kbps}$ and total data transfer of 6.7 MB.

2) Vehicles moving in opposite directions: Bandwidth and signal strength for vehicles moving in opposite direction under LOS and NLOS conditions are shown in Figs. $4 \mathrm{~g}$ and $4 \mathrm{~h}$ respectively. The average bandwidth for LOS and NLOS V2I is also shown for reference. The distance between the two vehicles is indicated on the $\mathrm{X}$-axis labels, such that the vehicles cross each other at the center of the graph. The same behavior of Wi-Fi RSSI as in V2V is seen here where it increases as vehicles come close to each other but the bandwidth does not increase with RSSI as we saw before because of the limiting effect of WiMAX.

In the crossing V2V2I setup on the LOS route an average relative vehicle speed of $58 \mathrm{~km} / \mathrm{h}$ was recorded with an average distance from the base station of $441 \mathrm{~m}$. The contact time of 36 seconds produced an average bandwidth of $454 \mathrm{kbps}$, jitter of $10.3 \mathrm{~ms}$ and total data transfer of $1.83 \mathrm{MB}$. The bandwidth starts lower, but quickly stabilizes as soon as the link is established between the two vehicles. The bandwidth peaks at the same value as with V2I, where only WiMAX is used; this shows that when within $\mathrm{Wi}-\mathrm{Fi}$ range, the performance depends on the WiMAX connection.

Under NLOS the signal strength is not stable as in LOS where the communication range is short, due to the vehicle BS separation and presence of obstacles that continually affect the signal. The resulting bandwidth is accordingly unstable. Even under unstable conditions of signal strength the bandwidth on average still matches that seen in the V2I test. The experiment was carried out under the average vehicular relative speed of $55 \mathrm{~km} / \mathrm{h}$ and average maximum separation of $192 \mathrm{~m}$ was reached. The average contact lasted for 35 seconds with an average bandwidth of $451 \mathrm{kbps}$ producing total data transfer of $1.82 \mathrm{MB}$ and $13.9 \mathrm{~ms}$ jitter.

The average values measured in all experiments performed are shown in Table I. For V2V communication where Wi-Fi was used, an increase in bandwidth is visible when vehicles follow each other than when moving in opposite directions. Since this is an average value for the entire contact period, 33 seconds, the bandwidth is lower when moving at opposite directions because at the edge of communication range the signal strength is weak hence lower bandwidth. The same behavior is experienced for V2V2I even though here the difference is not much due to the low bandwidth limitation induced by WiMAX. We also observe that the bandwidth for WiMAX in V2I is not significantly different to that in V2V2I for vehicles following and moving in opposite directions scenarios, both LOS and NLOS conditions.

Table I also gives the average jitter incurred for each test. In $\mathrm{V} 2 \mathrm{~V}$ communication where IEEE $802.11 \mathrm{n}$ is used, we see a lower jitter when cars are following each other than when they are moving in opposite directions. For the V2I case where only WiMAX is used we see an increased jitter compared to $\mathrm{V} 2 \mathrm{~V}$ where $\mathrm{Wi}-\mathrm{Fi}$ was used. We also observe an increase in jitter when operating under NLOS conditions due to the signal being reflected and weakened by blocking buildings and other objects. We further see an increased jitter in V2V2I when both Wi-Fi and WiMAX operate together. Similar to the results reported in [2, 21, 32], vehicle speed does not seem to impact bandwidth and jitter but rather the contact time. Distance between the communicating vehicles on the other hand, no matter their direction of movement, impacts bandwidth and jitter.

In addition to the results above, we successfully performed with good quality transmission:

- V2V2I: audio streaming (internet radio) at $64 \mathrm{kbps}$

- V2I and V2V2I: LD video streaming at $250 \mathrm{kbps}$, 
TABLE I: Wi-Fi and WiMAX performance in different VANET architectures.

\begin{tabular}{|c|c|c|c|c|c|c|c|c|c|}
\hline & & \multicolumn{2}{|c|}{$\mathrm{V} 2 \mathrm{~V}$} & \multicolumn{2}{|c|}{ V2I } & \multicolumn{4}{|c|}{ V2V2I } \\
\hline \multirow{2}{*}{\multicolumn{2}{|c|}{ Average values }} & \multirow[t]{2}{*}{ Crossing } & \multirow[t]{2}{*}{ Following } & \multirow[t]{2}{*}{ LOS } & \multirow[t]{2}{*}{ NLOS } & \multicolumn{2}{|c|}{ Crossing } & \multicolumn{2}{|c|}{ Following } \\
\hline & & & & & & LOS & NLOS & LOS & NLOS \\
\hline \multicolumn{2}{|c|}{ Jitter (ms) } & 1.88 & 0.38 & 8.22 & 8.56 & 10.3 & 13.9 & 10.2 & 12.4 \\
\hline \multicolumn{2}{|c|}{ Speed $(\mathrm{km} / \mathrm{h})$} & 64 & 113 & 31 & 33 & 58 & 55 & 27 & 25 \\
\hline \multirow{2}{*}{$\begin{array}{l}\text { Separation } \\
(\mathrm{m})\end{array}$} & Wi-Fi & 302 & 34 & - & - & 251 & 192 & 31 & 40 \\
\hline & WiMAX & - & - & 443 & 802 & 441 & 821 & 383 & 778 \\
\hline \multirow{2}{*}{$\begin{array}{c}\text { Bandwidth } \\
\text { (Mbps) }\end{array}$} & Avg. & 13.7 & 31.3 & 0.521 & 0.518 & 0.454 & 0.451 & 0.539 & 0.543 \\
\hline & Max. & 31.7 & 34.5 & 0.62 & 0.62 & 0.551 & 0.598 & 0.781 & 0.861 \\
\hline \multicolumn{2}{|c|}{ Contact Time (s) } & 33 & 100 & 100 & 100 & 36 & 35 & 100 & 100 \\
\hline \multicolumn{2}{|c|}{ Data Transferred (MB) } & 51.7 & 386.3 & 6.35 & 6.33 & 1.83 & 1.82 & 6.64 & 6.70 \\
\hline
\end{tabular}

- V2I and V2V2I: Skype voice call at $96 \mathrm{kbps}$,

- V2I and V2V2I: Skype video call at $250 \mathrm{kbps}$, and

- V2V: HD video streaming at 1.2 Mbps.

\section{CONCLUSION}

Feasibility of infotainment applications in vehicular ad-hoc networks depends not only on vehicular network characteristics but as well as the communication medium in terms of its performance under such networks. In this research, the performance of $\mathrm{Wi}-\mathrm{Fi}$ as a provider of inter-vehicular communications and WiMAX for vehicle to infrastructure communications in a simple vehicular ad-hoc network was evaluated. Experiments in scenarios with representative vehicle speeds, levels of urbanization, contact ranges and contact durations were conducted. Wi-Fi, used for the $\mathrm{V} 2 \mathrm{~V}$ connection, was found to provide reliable and high bandwidth, while connected. The Wi-Fi connection was unaffected by speed, and the only distinguishable factor seems to be separation which determined whether the connection is made. Wi-Fi ranges of up to 300 meters were achieved with external antennas. WiMAX bandwidth, used for V2I communications, is severely affected by even slight mobility. This is due to the $802.16 \mathrm{~d}$ standard, which is designed for fixed wireless communication. Once mobility is introduced error rate increases, therefore forcing the use of less aggressive modulation techniques. The WiMAX bandwidth is predictable and stable for the vehicular speeds tested, but mobile bandwidth is a fraction of the stationary bandwidth. The WiMAX bandwidth fluctuates for NLOS, but does not significantly affect the average bandwidth.

\section{REFERENCES}

[1] S. Olariu and M. C. Weigle, Vehicular Networks from Theory to Practice, COMPUTER and INFORMATION SCIENCE SERIES, 2009

[2] G. Marcelo, B. Fehmi, D. Marcelo, R. Savio, A. Rafael, H. Luis, C. Otto and E. Miguel, Measuring the Capacity of In-Car to In-Car Vehicular Networks, IEEE Communications Magazine, Nov. 2009, pp 128-136.

[3] J. Miller, Vehicle-to-Vehicle-to-Infrastructure (V2V2I) Intelligent Transportation System Architecture, IEEE Intelligent Vehicles Symposium, June 2008, pp 715-720.

[4] M. Raya and J. P. Hubaux, The Security of Vehicular Ad Hoc Networks, ACM SASAN, 2005.

[5] H. Hartenstein, H. Fuler, M. Mauve and W. Franz, Simulation Results and Proof-of-Concept Implementation of the FleetNet Position-Based Router, Personal Wireless Communications (PWC 2003), Venice, Italy, Sept. 2003, pp. 192197.

[6] C. Tchepnda, H. Moustafa, H. Labiod and G. Bourdon, Securing Vehicular Communications: An Architectural Solution Providing a Trust Infrastructure, Authentication, Access Control and Secure Data Transfer, ACM Autonet in conjunction with Globecom 2006.
[7] K. Lee, S.-H. Lee, R. Cheung, U. Lee, and M. Gerla, First experience with Cartorrent in a Real Vehicular ad hoc Network Test-bed, Mobile Networking for Vehicular Environments, 2007, pp. 109114.

[8] U. Varsheney, Vehicular Mobile Commerce, IEEE Computer Magazine, Dec. 2004.

[9] J. Tian and K. Rothermel, Building Large Peer-to-Peer Systems in Highly Mobile ad hoc Networks: New Challenges, Technical Report 2002/05, University of Stuttgard, 2002.

[10] M.J. Booysen, S. Zeadally, G.-J. van Rooyen, Survey of media access control protocols for vehicular ad hoc networks, IET Communications, vol.5, issue 11, July 2011.

[11] C. Wang, X. Cheng and D. Laurenson, Vehicle-to-Vehicle Channel Modelling and Measurements: Recent Advances and Future Challenges, IEEE Communications Magazine, Nov. 2009, pp 96-103.

[12] IEEE P802.11p: Draft Amendment to standard for Information technology - Telecommunications and information exchange between systemsLAN/MAN Specific RequirementsPart 11: Wireless LAN Medium Access Control (MAC) and Physical Layer (PHY) specifications: Wireless Access in Vehicular Environments (WAVE), 2007/2010.

[13] IEEE Standard for Information technologyTelecommunications and information exchange between systemsLocal and metropolitan area networksSpecific requirements Part 11: Wireless LAN Medium Access Control (MAC) and Physical Layer (PHY) Specifications, 2007.

[14] IEEE Standard for Information technologyTelecommunications and information exchange between systemsLocal and metropolitan area networksSpecific requirements Part 11: Wireless LAN Medium Access Control (MAC) and Physical Layer (PHY) Specifications, 2009.

[15] IEEE Standard for Local and metropolitan area networks Part 16: Air Interface for Broadband Wireless Access Systems, 2009.

[16] CarTalk 2000 Project. http://www.cartalk2000.net accessed 27 Jun. 11.

[17] CARLINK. CARLINK D2.1 - Architecture definition. Chapter 1: Physical platform overview. CARLINK consortium, September 2007. http://carlink.lcc.uma.es/ accessed 27 Jun. 11.

[18] A. Festag, G. Noecker, M. Strassberger, A. Lbke, B. Bochow, M. Torrent-Moreno, S. Schnaufer, R. Eigner, C. Catrinescu, and J. Kunisch, NoW Network on Wheels: Project Objectives, Technology and Achievements, Proceedings of 5rd International Workshop on Intelligent Transportation (WIT), Hamburg, Germany, March 2008, pp. 211-216: http://www.network-on-wheels.de/documents.html accessed 27 Jun. 11.

[19] Car2Car Communication Consortium Manifesto v1.1, Overview of the C2C-CC System, August 2007.

[20] A. Tufail, M. Fraser, A. Hammad, K. Ki Hyung and S. Wha Yoo, An Empirical Study to Analyze the Feasibility of WIFI for VANETs, IEEE Communications Magazine, April 2008, pp 553-558.

[21] M. Wellens, B. Westphal, and P. Mahonen, Performance Evaluation of IEEE 8002.11-based WLANs in Vehicular Scenarios, in IEEE VTC 2007, April 2007, pp 1167-1171.

[22] C. Chou, C. Li, W. Chien, and K. Lan, A Feasibility Study on Vehicleto-Infrastructure Communication: Wi-Fi vs. WiMAX, In Proc. of the Tenth International Conference on Mobile Data Management: Systems, Services and Middleware, 2009. MDM09, May 2009, pp 397398.

[23] I. Msadaa, P. Cataldi, and F. Filali, A Comparative Study between 802.11p and Mobile WiMAX-based V2I Communication Networks, Fourth International Conference on Next Generation Mobile Applications, Services and Technologies (NGMAST), July 2010, pp 186191.

[24] J. Eriksson, H. Balakrishnan and S. Madden, Cabernet: Vehicular Content Delivery Using WiFi, ACM, Mobicom 08, September 2008. 